\title{
Horner's Syndrome Secondary to Epidural Anaesthesia Following Posterior Instrumented Scoliosis Correction
}

\author{
Simon Cowie ${ }^{1}$, Lucinda Gunn ${ }^{2}$, Pradeep Madhavan ${ }^{1}$ \\ ${ }^{1}$ Spinal Orthopaedic, Musgrove Park Hospital, Taunton \& Somerset NHS Trust, Taunton, UK \\ ${ }^{2}$ Medical Directorate, Bristol Royal Infirmary, Bristol, UK
}

An 11-year-old girl underwent T4 to L1 posterior instrumented scoliosis correction for adolescent idiopathic scoliosis. Postoperative clinical examination revealed left-sided Horner's syndrome which was preceded by left-sided C8 paraesthesia. The Horner's syndrome resolved after 14 hours following weaning and removal of the epidural catheter. Horner's syndrome following posterior instrumented scoliosis correction associated to epidural use is extremely rare. Surgeons must be aware of the risks of epidural placement and the need for close monitoring of associated complications. Alternative aetiology producing a Horner's syndrome must always be considered because of its devastating long term sequela if missed.

Keywords: Epidural; Horner syndrome; Scoliosis

\section{Introduction}

Horner's syndrome following posterior instrumented scoliosis correction associated to epidural use is extremely rare. Surgeons must be aware of the risks of epidural placement and the need for close monitoring of associated complications. We aim to outline the potential clinical and surgical manifestations of Horner's syndrome, summarize its pathophysiology and discuss investigations and management to enhance awareness and improve patient safety and care.

\section{Case Report}

A 11-year 4 month old girl underwent posterior scoliosis correction for Lenke $1 \mathrm{CN}$ scoliosis deformity with mild thoracic hypokyphosis, $56^{\circ}$ right-sided thoracic curve centred on $\mathrm{T} 8 / \mathrm{T} 9,48^{\circ}$ proximal thoracic curve centred on T3, and $28^{\circ}$ lumbar curvature centred on L2 and Risser grade zero (Figs. 1, 2) [1]. Preoperative consultant paediatric and anaesthetic assessments revealed no significant clinical findings and subsequent lung function tests were normal.

T4 to L1 posterior instrumented fusion was completed under general anaesthesia. Grade 1 laryngoscopy was performed using a size 6.0 endotracheal tube. Propofol, atracurium, and fentanyl were used at induction with an additional $4 \mathrm{mg}$ of dexamethasone. Peripheral access and arterial lines were sited; no central venous access was required. The eyes were protected with lubricant and taped

Received Mar 9, 2014; Revised Jun 12, 2014; Accepted Jun 12, 2014

Corresponding author: Simon Cowie

Spinal Orthopaedic, Musgrove Park Hospital, Taunton, Somerset, [TA1 5DA], UK

Tel: +44-01823-333444, Fax: +44-01823-343444, E-mail: simon.cowie84@gmail.com 
shut. Face and eye positioning were checked in the mirror of the Proneview (Mizuho OSI, Union City, CA, USA) after turning the patient prone onto a Jackson table with the abdomen free. The patient's neck was maintained in a neutral position throughout the procedure with shoulders

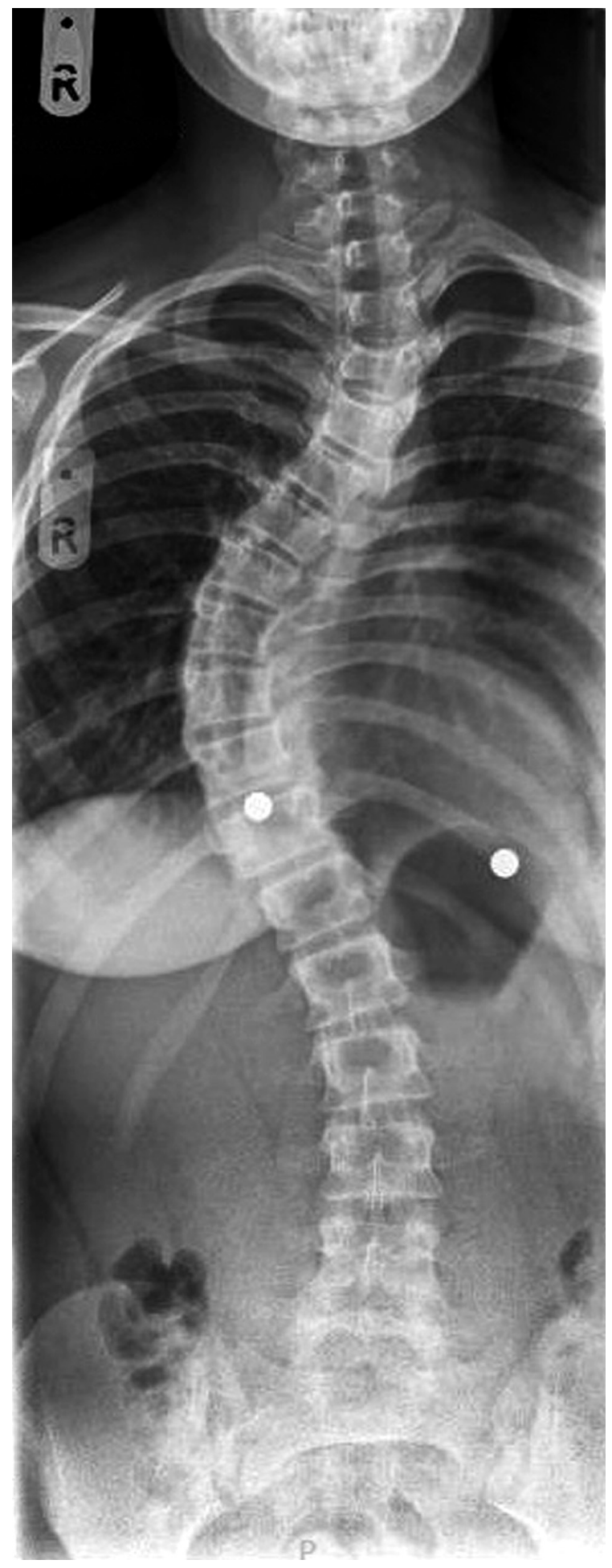

Fig. 1. Preoperative anteroposterior whole spine. in abduction. Pressure points were protected and checked after the procedure.

Anaesthesia was maintained for 5 hours and 50 minutes using positive pressure ventilation and oxygen. Remifentanil was commenced 1 hour into the procedure

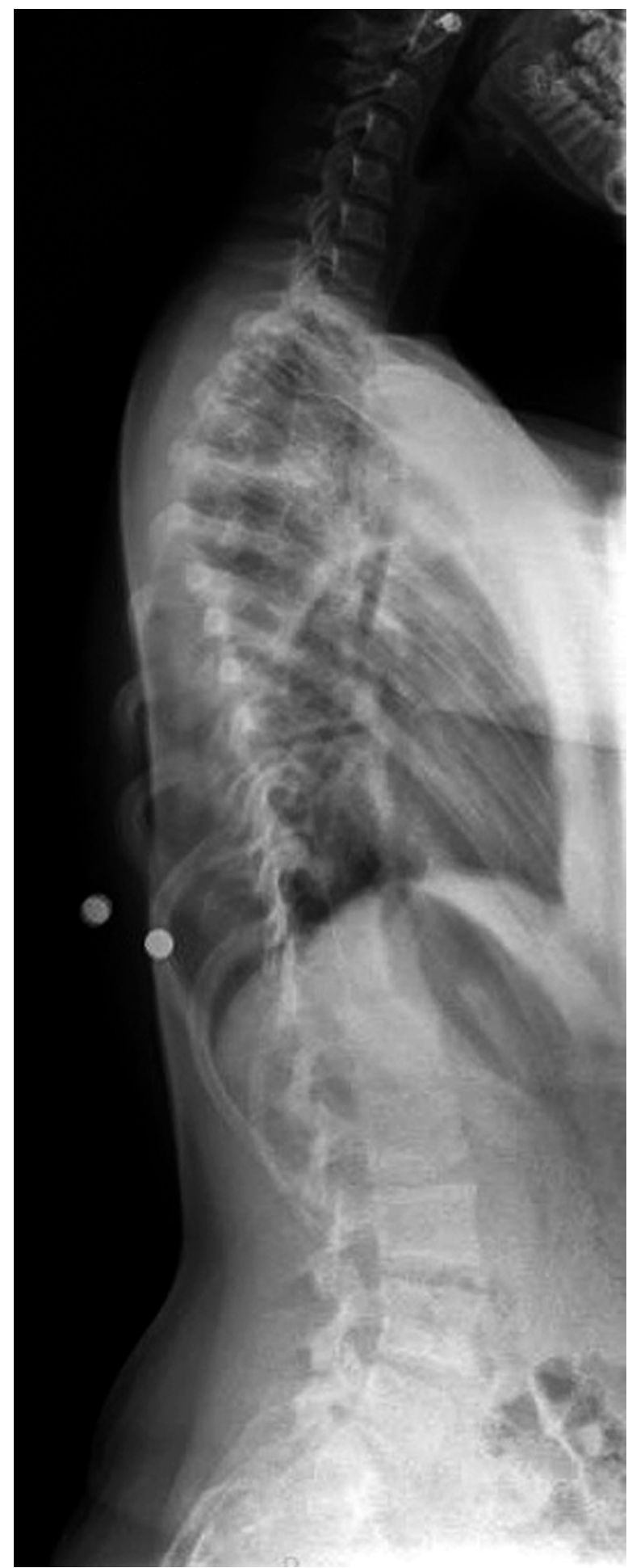

Fig. 2. Preoperative lateral whole spine. 
along with propofol infusion, $2 \mathrm{~g}$ of magnesium sulphate was given at $1 \frac{1 / 2}{2}$ hours followed by $20 \mathrm{mg}$ of ketamineat 2

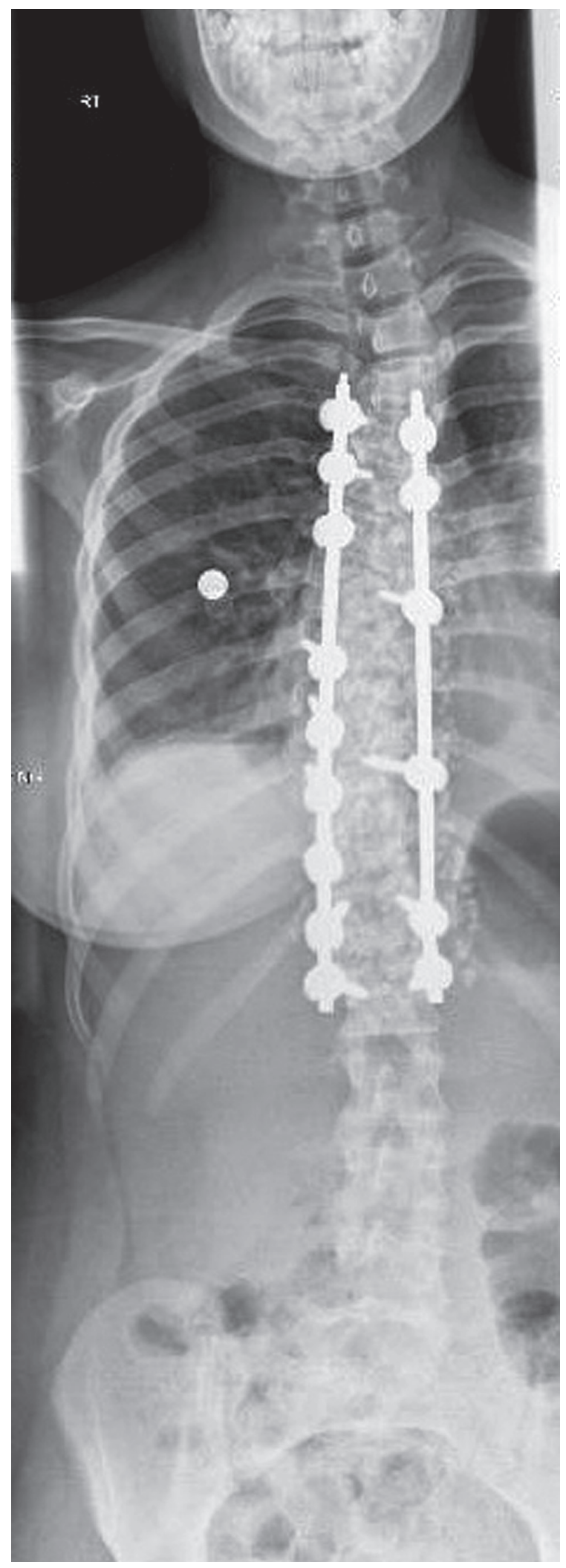

Fig. 3. Postoperative anteroposterior whole spine.
$1 / 2$ hours. Systolic blood pressure was maintained between 80-95 with patient warming to maintain a core body temperature of between $36.9^{\circ} \mathrm{C}$ and $37.6^{\circ} \mathrm{C}$ throughout.

Intraoperatively $16 \mathrm{~mL}$ of $0.5 \%$ bupivacaine with adrenaline (1 in 200,000) was injected prior to skin incision. The surgery was uncomplicated; pedicle screws were placed in a box configuration at T4, T5, T10, T12, and L1. Further pedicle screws were sited alternately in all vertebrae in between (Figs. 3, 4). Screw placement and correction was

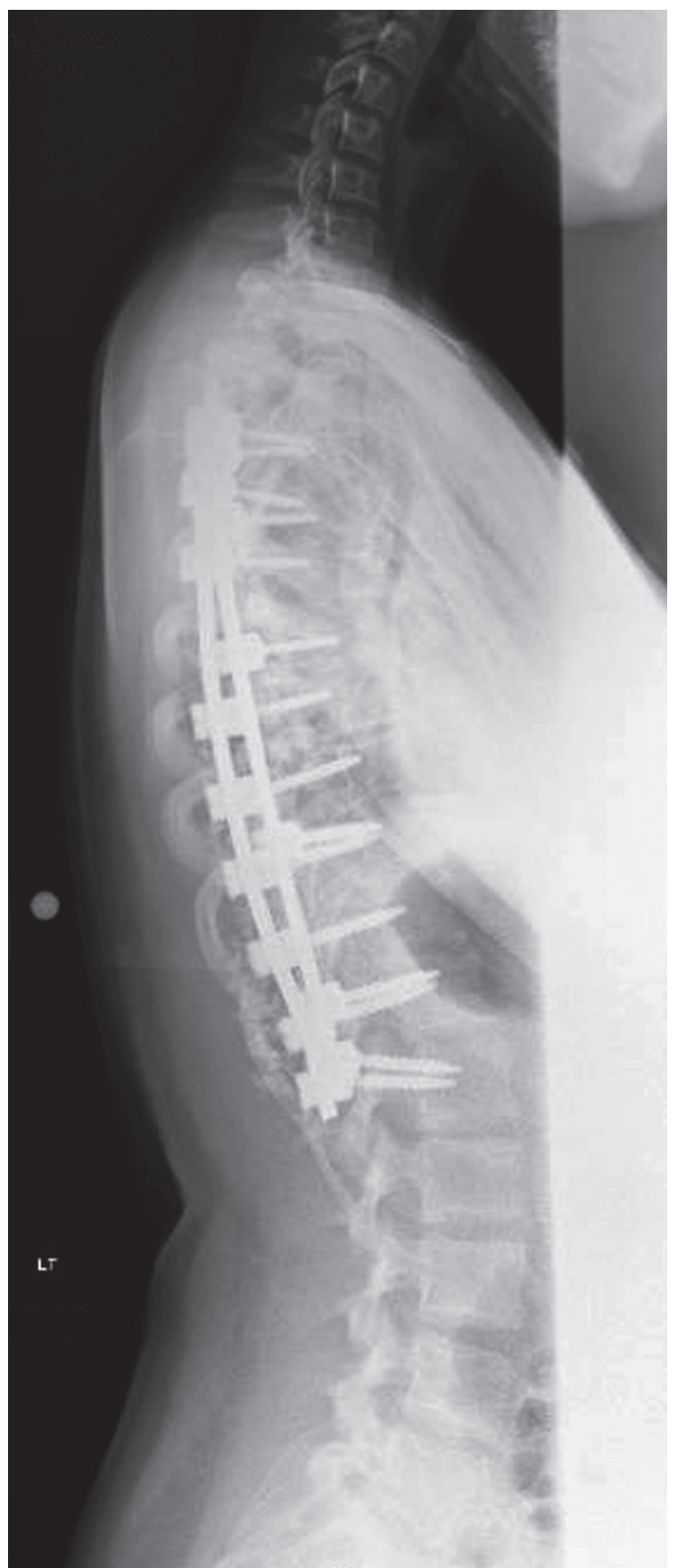

Fig. 4. Postoperative lateral whole spine. 
performed under intraoperative image control, and no mal-placement/breach was identified. Thoracic pedicle hook placement was not performed, and osteotomy was not required. Spinal cord monitoring (motor evoked potential/somatosensory evoked potential) was normal throughout. An excellent correction was achieved using contoured cobalt chrome rods. The spine was decorticated. Total blood loss was $600 \mathrm{~mL}$ and $127 \mathrm{~mL}$ was reinfused via cell salvage (haematocrit $48 \%$ ).

An epidural catheter was placed under direct vision at T9 and directed $5 \mathrm{~cm}$ proximally. This was uncomplicated and no blood or cerebrospinal fluid was aspirated. At the end of the procedure a bolus of $20 \mathrm{~mL} 0.125 \%$ plain bupivacaine was directly administered into the epidural catheter. Extubation and immediate recovery were uncomplicated.

A levobupivacaine $(0.1 \%)$ and fentanyl $(2 \mu \mathrm{g} / \mathrm{mL})$ epidural was delivered for analgesia. A patient-controlled analgesia pump with morphine was available along with regular oral paracetamol and diclofenac.

Five hours postoperatively, the patient complained of paraesthesia with a left C8 distribution. The epidural was reduced from 20 to $7 \mathrm{~mL} / \mathrm{hr}$, and symptoms resolved spontaneously. Over the following 24 hours the epidural rate was $8-13 \mathrm{~mL} / \mathrm{hr}$. At 24 hours postoperatively the patient developed unilateral left-sided miosis and ptosis. There was no associated headache, visual or psychological disturbance. The patient had been consistently haemodynamically stable; the Glasgow Coma Scale was, eyes 4, verbal 5, and motor 6. Pupils measured $5 \mathrm{~mm}$ (right) and $3 \mathrm{~mm}$ (left) with normal light reflexes. Fundoscopy was normal and peri-orbital swelling was absent. Pharmacological testing was not carried out. A comprehensive cranial and peripheral neurological examination revealed no further deficit.

A diagnosis of Horner's syndrome was made based on clinical examination findings. Accordingly, the epidural rate was decreased by $2-3 \mathrm{~mL} / \mathrm{hr}$ and weaned to removal within 16 hours. Following weaning, the Horner's syndrome resolved within 14 hours of onset with no residual deficit. Further invasive investigations and imaging were therefore deemed unnecessary. No cardiorespiratory compromise occurred and total spinal anaesthesia was not noted. Controlled, monitored weaning therefore allowed adequate analgesic cover. No specific follow up was required for the Horner's syndrome. Routine scoliosis correction was followed up at 3, 6, and 12 months.

\section{Discussion}

Horner's syndrome related to epidural anaesthesia is rare $(0.4 \%-2.5 \%)$ [2]. The syndrome is typically recognised as a triad of ipsilateral signs, that is, ptosis, miosis and anhydrosis. This syndrome develops due to disruption within the oculosympathetic pathway which can occur at different levels. Pathway fibres arise from the posterolateral hypothalamus and descend uncrossed in the lateral portion of the midbrain, pons, medulla and cervical spinal cord to reach the ciliospinal centre at the $\mathrm{C} 8-\mathrm{T} 2$ level [3] (occasionally as low as T4) [4]. Here they synapse with preganglionic fibres. Preganglionic (second order) fibres exit the cord via the anterior rami and travel cranially in the sympathetic chain over the apex of the lungs and through the stellate and inferior cervical ganglion passing around the subclavian artery and through the middle cervical ganglion to the superior cervical ganglion at the carotid bifurcation. Here they synapse with postganglionic (third order) neurones. The fibres then remain in close proximity to the internal carotid artery to supply the pupillary muscles, conjunctiva, levator palpebrae and sweat glands of the face.

First order neuron disorders are associated with central pathology within the hypothalamospinal tract. Second and third order neuron disorders involve interruption of the sympathetic outflow tract. Pre versus post ganglionic lesions can be tested and classified using hydroxyamphetamine [5] and further information can be gained by computed tomography/magnetic resonance imaging/ magnetic resonance angiography scanning. Disruption to postganglionic fibres can present as partial Horner's syndrome with anhydrosis absent or limited to the middle portion of the forehead. Vasodilatation may lead to flushing of the affected side of the face. Pupillary light reflex is maintained as this is under parasympathetic control.

Epidural anaesthesia is a central neuroaxial block technique. The segmental nerves in the thoracic and lumbar region contain somatic sensory, motor, and autonomic nerve fibres. Sympathetic outflow extends from T1-L2. Sensory and autonomic fibres have smaller diameters and are more easily blocked than large rapidly-conducting motor fibres, and sympathetic blocks usually extend 1-2 levels higher than sensory blocks. An epidural sited at T9 would normally cause both sensory and motor blockade up to 2-3 segments above insertion. The locational variability of the ciliospinal centre C8-T2 (occasionally 
as low as T4) in combination with an inadvertent high blockade secondary to either excessively large doses of local anaesthetic or high spread due to posture, is an explainable cause of Horner's syndrome. Haemodynamic and respiratory stability indicate the blockade had not fully reached the $\mathrm{T} 2$ level despite transient $\mathrm{C} 8$ paresthesia of the left arm.

We note that although uncommon, many reports have been published in the Obstetrics and Gynaecology literature related to lumbar epidural placement in pregnancy, labour and caesarean sections causing Horner's syndrome. Anatomical and physiological changes during pregnancy and labour may explain the volume of reported to some degree, as changes in intra-abdominal pressure divert blood through the epidural venous plexus and subsequent reduced volume within the epidural space and increased pressures might lead to a more cranial distribution of epidural local anaesthetic [2,6-9].

Previous reports have cited a range of several minutes to 24 hours until resolution. In vitro studies may explain why sympathetic blockade can manifest several spinal segments above the level of infiltration. The sympathetic fibres at the cervico-thoracic level are of small diameter [10], and thus, are more sensitive to local anaesthesia. Unilateral symptoms may be secondary to the anatomical placement of an epidural catheter, septations within the epidural space and patient positioning in a lateral semirecumbent position producing more concentrated anaesthesia toward the dependent side.

The persistence of Horner's syndrome may warrant formal cranial, cervical and vascular imaging. The literature highlights cases with symptoms persisting up to 6 months from original insult [11]. We note a case report by Mueller et al. [12] who described Horner's syndrome after scoliosis correction without the use of epidural anaesthesia that was related to hook placement around the T4 pedicle. Early follow up of patients with no or partial resolution of symptoms is required to manage the potential long term sequela. Under certain circumstances these may require surgical intervention.

It is likely the effect of epidural local anaesthetic on the sympathetic nervous system was the cause of Horner's syndrome in our patient, as was suggested by the lack of intra-operative complications, cardiorespiratory compromise coupled with delayed onset of blockade symptoms, and complete resolution on epidural weaning.

However, alternative aetiologies must always be con- sidered. Excepting congenital/neurological associations, plausible considerations following scoliosis correction could include: (1) Sympathetic blockade related to epidural usage, (2) Direct insult to the sympathetic chain at the level of the neck or blunt/penetrating trauma from surgical intervention in the thoracic region [12], (3) Compression of the sympathetic chain by e.g., haematoma related to surgery, (4) Dissecting thoracic aortic aneurysm or carotid artery dissection, (5) Cavernous sinus thrombosis, 6) Tube thoracostomy (when an anterior approach is used).

We highlight the importance of including epidural anaesthesia within the differential diagnosis of acute Horner's syndrome. We advocate the placement of the epidural catheter intraoperatively under direct vision. Basic management must be meticulous and include monitoring of patient position and epidural infusion rate. Reduction of the epidural rate should be the first intervention if Horner's syndrome develops. Thorough neurological and ophthalmology review is advocated and intra-operative technique and surgical complications should be examined and considered as potential causes.

\section{Conflict of Interest}

No potential conflict of interest relevant to this article was reported.

\section{References}

1. Lenke LG, Betz RR, Harms J, et al. Adolescent idiopathic scoliosis: a new classification to determine extent of spinal arthrodesis. J Bone Joint Surg Am 2001;83:1169-81.

2. Clayton KC. The incidence of Horner's syndrome during lumbar extradural for elective Caesarean section and provision of analgesia during labour. Anaesthesia 1983;38:583-5.

3. Kerr RW, Brown JA. Pupillomotor pathways in the spinal cord. Arch Neurol 1964;10:262-70.

4. Ray BS, Hinsey JC, Geohegan WA. Observations on the distribution of the sympathetic nerves to the pupil and upper extremity as determined by stimulation of the anterior roots in man. Ann Surg 1943;118:64755.

5. Van der Wiel HL, Van Gijn J. Localization of Horner's syndrome. Use and limitations of the hydroxyamphetamine test. J Neurol Sci 1983;59:229-35. 
6. Jeret JS, Mazurek AA. Acute postpartum Horner's syndrome due to epidural anesthesia. Arch Ophthalmol 1995;113:560.

7. Biousse V, Guevara RA, Newman NJ. Transient Horner's syndrome after lumbar epidural anesthesia. Neurology 1998;51:1473-5.

8. Lynch JH, Keneally RJ, Hustead TR. Horner's syndrome and trigeminal nerve palsy following epidural analgesia for labor. J Am Board Fam Med 2006;19:521-3.

9. Chandrasekhar S, Peterfreund RA. Horner's syndrome following very low concentration bupivacaine infusion for labor epidural analgesia. J Clin Anesth 2003;15:217-9.

10. Fish RE. Anesthesia and analgesia in laboratory animals. Boston: Elsevier/Academic Press; 2008.

11. Hered RW, Cummings RJ, Helffrich R. Persistent Horner's syndrome after spinal fusion and epidural analgesia. A case report. Spine (Phila Pa 1976) 1998;23:387-90.

12. Mueller KL, Loder RT, Eggenberger ER, Farley FA. Horner's syndrome after posterior spinal fusion in a child: a case report. Spine (Phila Pa 1976) 2000;25:2836-7. 